\title{
Experimental and numerical study of heat flux in dual bell nozzles
}

\author{
Chloé Génin, Andreas Gernoth ${ }^{\dagger}$ and Ralf Stark ${ }^{\ddagger}$ \\ German Aerospace Center, Lampoldshausen, D-74239, Germany
}

\begin{abstract}
The characteristic contour inflection of a dual bell nozzle is the key to altitude adaption. In sea level conditions, it forces the flow to a symmetrical separation, limiting the side load generation and increasing the thrust. After the transition, under high altitude conditions, the nozzle flows full, increasing the vacuum thrust. A hot flow experimental study has been conducted at the German Aerospace Center on a planar dual bell nozzle. The wall temperature distribution has been measured at various depths for the determination of the heat flux through the wall. The region of the inflection is of particular interest for the adjustment of the conventional cooling methods of dual bell nozzles. The contour inflection leads to a local increase of the thermal loads. In addition to the tests, the flow behavior and thermal loads have been calculated with CFD method and compared with the experiment.
\end{abstract}

\section{Nomenclature}

$\begin{array}{ll}\alpha & \text { Inflection angle } \\ \epsilon & \text { Area ratio } \\ N P R & \text { Nozzle Pressure Ratio, } P_{0} / P_{a} \\ P & \text { Pressure, MPa } \\ q & \text { Local heat flux, } \mathrm{W}^{-2} \\ T & \text { Temperature, } \mathrm{K} \\ x, y & \text { Axial and radial coordinate, } \mathrm{mm} \\ \text { Subscript } & \\ 0 & \text { Total conditions } \\ a & \text { Ambient } \\ b & \text { Base nozzle } \\ e & \text { Extension nozzle } \\ w & \text { Wall }\end{array}$

\section{Introduction}

The European heavy lifter Ariane 5 features a parallel staged design, with a cryogenic main stage supported by two solid boosters generating the main part of the lift-off thrust. The main stage engine Vulcain 2 has to be ignited on the ground for security reasons. The proper running of the engine has to be assured before ignition of the solid boosters and take off of the rocket. Because of this constraint, the main stage engine has to fulfill a wide range of operation conditions, from sea level to almost vacuum. Flow separation in the nozzle has to be avoid at sea level to reduce the amplitude of undesired side loads, that may damage engine and payload. The resulting area ratio limitation leads to performance losses: at sea level, the engine exhaust flow is driven over expanded and at high altitude, highly under expanded.

Altitude adaptive nozzle concepts generate a thrust that is not only optimized for one specific altitude, increasing the overall specific impulse $I_{s p}$ of the engine during ascent. Therefore, they have come into focus as the sub system with the most promising performance gain. Different concepts were developed to circumvent

*Research scientist, Nozzle group, Institute of Space Propulsion.

${ }^{\dagger} \mathrm{PhD}$. student, Fluid and Structures group, Institute of Space Propulsion.

${ }^{\ddagger}$ Head of Nozzle group, Institute of Space Propulsion. 
the limitation in area ratio of conventional nozzles. The commonly discussed solutions are plug, extendible and dual bell nozzles.

The characteristic contour inflection of the dual bell nozzle divides the nozzle into base and extension (Fig. 1) and offers a one-step altitude adaptation. The concept exists in the literature since 1949, proposed in a study by Foster and Cowles, ${ }^{1}$ but the first investigations were conducted only in the early 1990's (for example by Horn and Fisher ${ }^{2}$ or Hagemann et al. ${ }^{3}$ ).
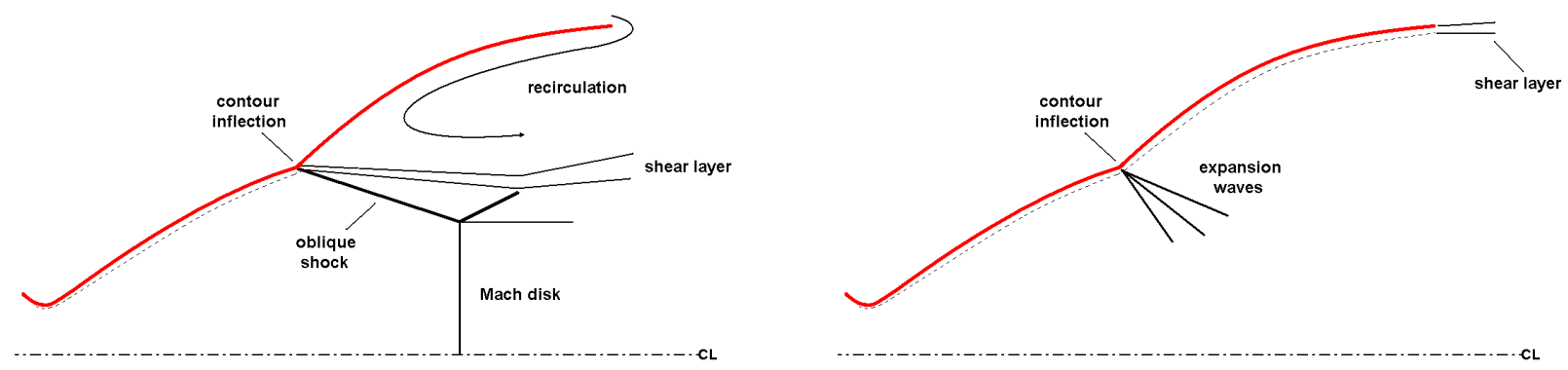

Figure 1. The two operation modes of a dual bell nozzle: sea level mode (left) and altitude mode (right).

Under sea level conditions, the flow separates at the contour inflection in a controlled and symmetrical way. The side load generation is kept reduced and the thrust increased due to the small area ratio. During flight, the ambient pressure decrease, increasing the nozzle pressure ratio $\left(\mathrm{NPR}=P_{0} / P_{a}\right)$. At a certain altitude, the transition NPR is reached and the separation point leaves the contour inflection and moves rapidly (in a few milliseconds) to the nozzle exit. The extension flows then full. The altitude thrust is increased due to the larger area ratio of the extension.

\section{Motivation}

The flow behavior in dual bell nozzles has been intensively investigated in various cold and hot flow experimental studies ${ }^{4}$ at the German Aerospace Center (DLR). Prior to hot firing tests of dual bell nozzles, its particular needs in cooling has to be known, and thus the thermal load variation due to the contour inflection.

A test campaign has been conducted on the test bench complex M11 at DLR Lampoldshausen. A subscale planar nozzle was designed and tested under various conditions in pressure and temperature. Temperature measurements made in the wall at various depths yield information about the thermal fluxes through the wall.

For a better understanding of the flow physics and thermal behavior, a numerical study has been conducted. The experiments were simulated using the commercial CFD tool Fluent. The numerical results are presented and compared with the test data for validation.

\section{Description of the experiment}

The test campaign has been conducted at the test complex M11 at the DLR Lampoldshausen. ${ }^{7}$ The position M11.1 features four oxygen-hydrogen burners. Pressurized air is heated by flowing through the burners and reaches total temperature up to $1500 \mathrm{~K}$. Depending on the operating point and the nozzle throat geometry, the maximal total pressure reached is 30 bar. For the operation points chosen in this study, the flow was composed of about $95 \%$ of air with $5 \%$ of water damp (depending on air, oxygen and hydrogen mass flows).

The test facility offers short test duration, between $5 \mathrm{~s}$ and $60 \mathrm{~s}$ in steady state. The nozzle model is mounted on a horizontal rig and tested under ambient conditions in temperature and pressure $\left(P_{a}=1\right.$ bar and $T_{a}=290 \mathrm{~K}$ ).

The model tested in this campaign was a planar nozzle to permit a direct optical access of the region of the contour inflection. The base nozzle was chosen as a non truncated ideal nozzle with a design Mach number $M_{D}=2.8$. The extension was designed on an isobar (constant pressure extension). The geometrical parameters and a sketch of the model are given in Fig. 2.

The design parameters correspond to a total temperature $T_{0}=1000 \mathrm{~K}$ and a total pressure of $P_{0}=$ 


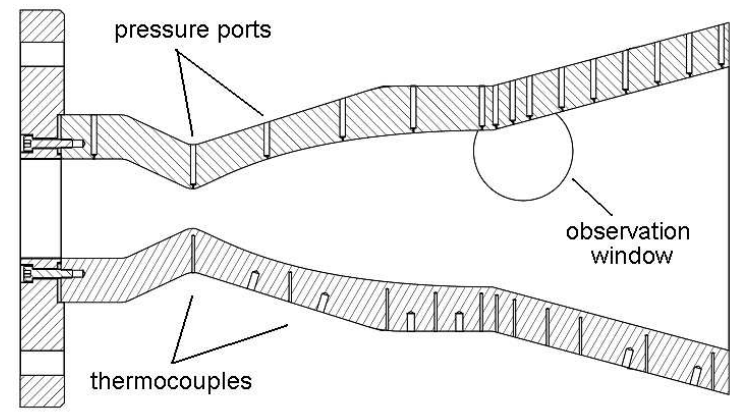

\begin{tabular}{lcc}
\hline Throat radius & $R_{t h}$ & $9 \mathrm{~mm}$ \\
Base length & $L_{b} / R_{t h}$ & 15.1 \\
Extension length & $L_{e} / R_{t h}$ & 11.9 \\
Area ratio & $\epsilon_{b}$ & 3.9 \\
& $\epsilon_{e}$ & 7.1 \\
Inflection angle & $\alpha$ & $15^{\circ}$ \\
Depth & $\mathrm{d}$ & $45 \mathrm{~mm}$ \\
\hline
\end{tabular}

Figure 2. Geometry of the planar dual bell model.

$3 \mathrm{MPa}$, for a nozzle tested under ambient conditions. The nozzle model was made of heat resistant steel with a wall thickness of $10 \mathrm{~mm}$ for the contour sides and the lateral plates.

Wall pressure and temperature measurements were conducted. The pressure ports were placed along the centerline of the nozzle contour. To avoid the necessity of active cooling, the pressure transducers were not placed directly in the nozzle wall. Small pipes were welded in the wall and linked with the transducers through Teflon tubes.

Temperatures were measured with thermocouples placed along the nozzle wall. They were positioned at $1 \mathrm{~mm}$ and $3 \mathrm{~mm}$ to the inner wall (hot flow side) at the same axial position. The sensor sensitivity is assured up to $1.5 \mathrm{~K}$ or $\pm 0.4 \%$ of the temperature.

\section{Numerical method}

The calculations have been conducted with the software package Ansys Fluent v12.1. The mesh was a hybrid non adaptive 2D grid (planar nozzle) with 164675 elements. The wall resolution auf $1 \mu \mathrm{m}$ led to a maximal value of $y^{+}=1.1$ for the 15 bar test case. The mesh for the solid body of the nozzle was a structured one. Figure 3 is an illustration of the grids: complete, magnified area of the nozzle body and nozzle wall.
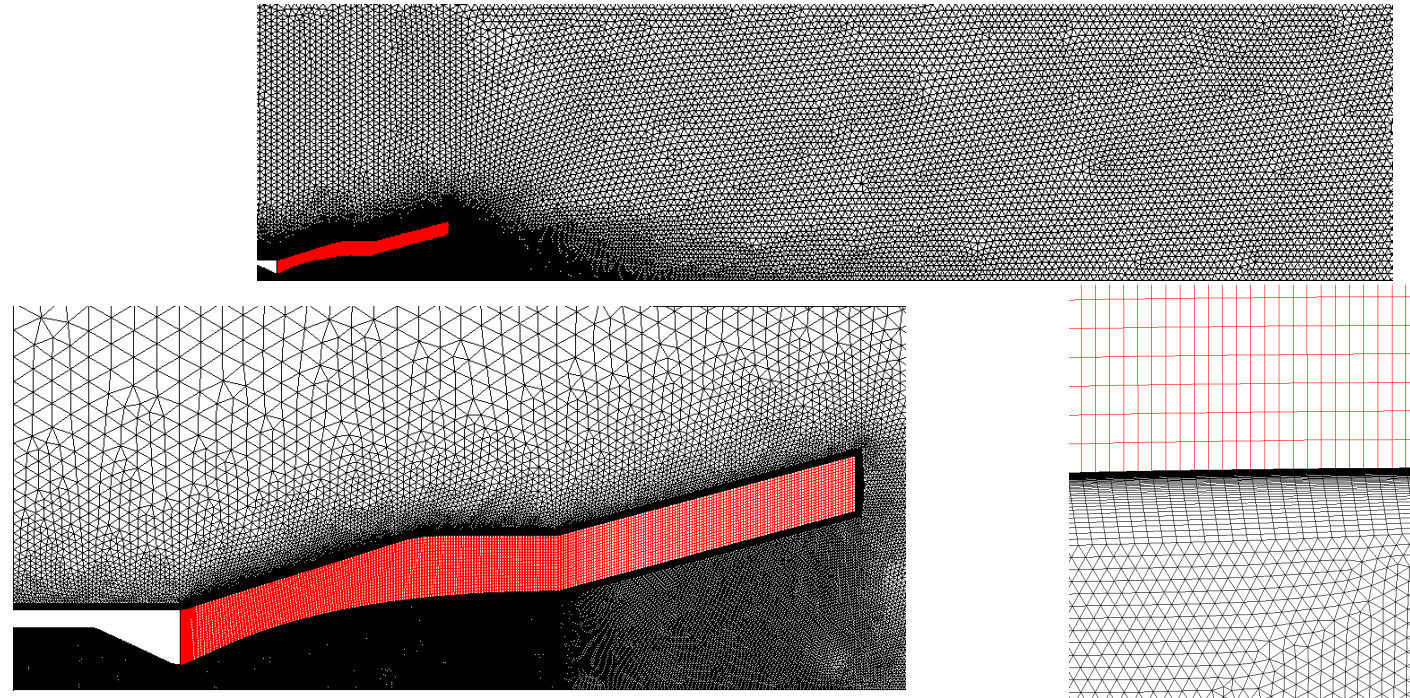

Figure 3. Nozzle geometry and calculation grid.

The test case has been first calculated in cold flow conditions to validate the numerical method. The boundary conditions are given in table 1 .

For the calculation of the cold flow test case, the solid body was not taken into account. The working fluid was pure nitrogen, taken as ideal gas with the following properties: $\mathrm{Cp}=1040.67 \mathrm{~J} / \mathrm{kg} . \mathrm{K}$, thermal 
Table 1. Boundary conditions for cold flow simulation.

\begin{tabular}{lcc}
\hline Inlet & total pressure & $10-32 \mathrm{bar}$ \\
& total temperature & $293.15 \mathrm{~K}$ \\
Farfield & temperature & $293.15 \mathrm{~K}$ \\
& Mach number & 0.01 \\
& pressure & $1 \mathrm{bar}$ \\
\hline
\end{tabular}

conductivity is $0.0242 \mathrm{~W} / \mathrm{mK}$, viscosity $1.663 e^{-5}$ Pas and molecular mass $28.0134 \mathrm{~kg} / \mathrm{kmol}$. The boundary conditions are summarized in table 2.

\begin{tabular}{ccc} 
Table 2. & Boundary conditions for warm & flow simulation. \\
\hline Inlet & total pressure & $15-30 \mathrm{bar}$ \\
& total temperature & $1000 \mathrm{~K}$ \\
& $\mathrm{H}_{2} \mathrm{O}$ (in mass fraction) & 0.28601 \\
& $\mathrm{O}_{2}$ (in mass fraction) & 0.06 \\
& $\mathrm{~N}_{2}$ (in mass fraction) & 0.65399 \\
Farfield & temperature & $293.15 \mathrm{~K}$ \\
& Mach number & 0.01 \\
& pressure & $1 \mathrm{bar}$ \\
& $N_{2}$ (in mass fraction) & 1 \\
\hline
\end{tabular}

For the warm flow simulation, the fluid was calculated as multi species. It is composed of nitrogen, oxygen and water damp, which all are described as ideal gas. Each of the material properties are modeled as temperature dependant.

The solid body was made of heat resistant steel Ferrotherm, whose thermal conductivity also depends on the temperature. The planar density based solver of Fluent was applied for the calculations. The original Spalart-Allmaras model ${ }^{5}$ has been chosen as turbulence model for its good results in the calculation of nozzle flow separation. ${ }^{6}$ A second order method was implemented for the calculation of the flow and the turbulence for the cold and warm flow test cases.

\section{Results and discussion}

\section{V.A. Nozzle flow behavior}

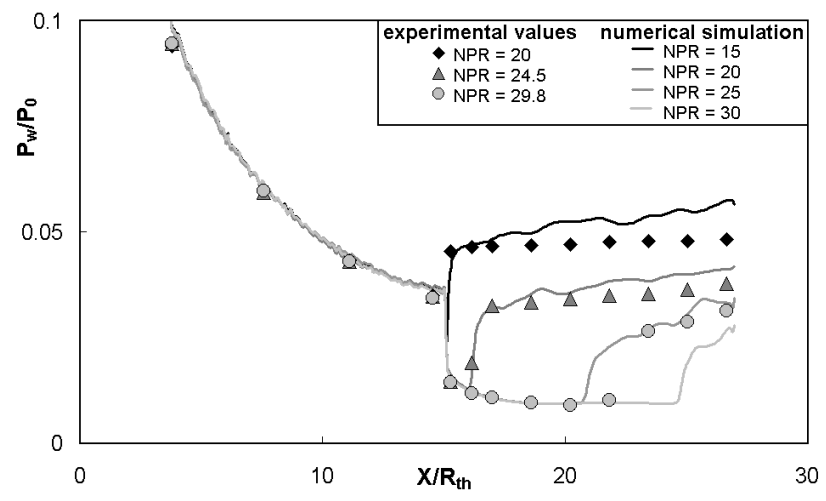

Figure 4. Wall pressure repartition for various NPR values.

The wall pressure has been measured along the nozzle contour for various values of NPR and total 
temperature. Figure 4 represents the pressure distribution for different conditions in pressure with the same temperature $T_{0}=1000 \mathrm{~K}$.

For low value of the NPR (here NPR $=20$ ), the flow is attached in the base nozzle and separates at the contour inflection. The nozzle is operating in sea level mode. When increasing the NPR value (NPR $=24.5$ ), the separation point leaves the inflection and starts moving down the extension. The first region of the extension features a strong negative pressure gradient and is designated as the inflection region. The sneak transition corresponds to the displacement of the separation point in the inflection region and ends with the start of the actual transition.

The design method for a constant pressure extension leads in planar nozzle to a slight negative wall pressure gradient in the extension, due to three dimensional effects in the flow. The expected transition does not take place and the separation point moves instead progressively down the extension $(\mathrm{NPR}=29.8)$. Altitude mode is reached only when the flow is attached down to the end of the extension.

The calculated pressure distribution is given for comparison. In sea level mode, the values show a very good accordance. For higher NPR values, the position of the calculated separation point is further down stream as the measured one.
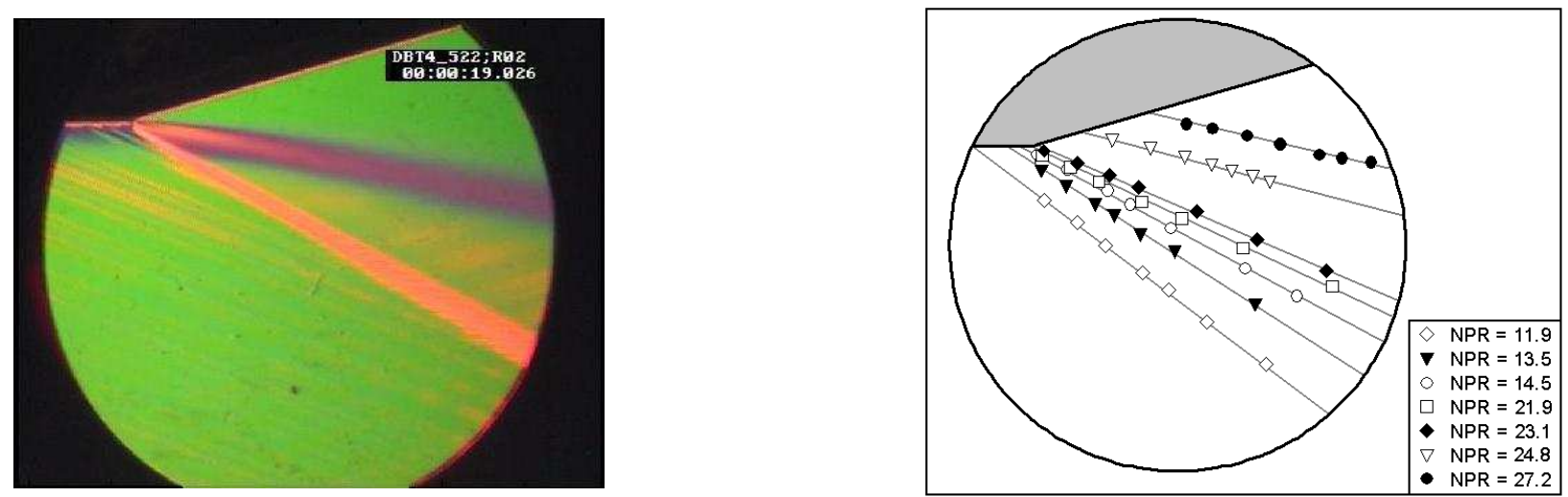

Figure 5. Schlieren picture of the flow in the inflection region (left) and angle of the separation shock for various NPR values (right).

In addition to the presented campaign, a series of cold flow tests have been conducted during which the flow has been observed using schlieren optics. Circular windows in the vicinity of the contour inflection permit to record the evolution of the shock system when varying the NPR value. Figure 5 (left) is a picture taken during sea level mode. The position and the shape of the separation shock can be taken out of the schlieren images and serve as a comparison for numerical validation. The separation shock in the vicinity of the inflection is represented in Fig. 5 (right) for the experiment.
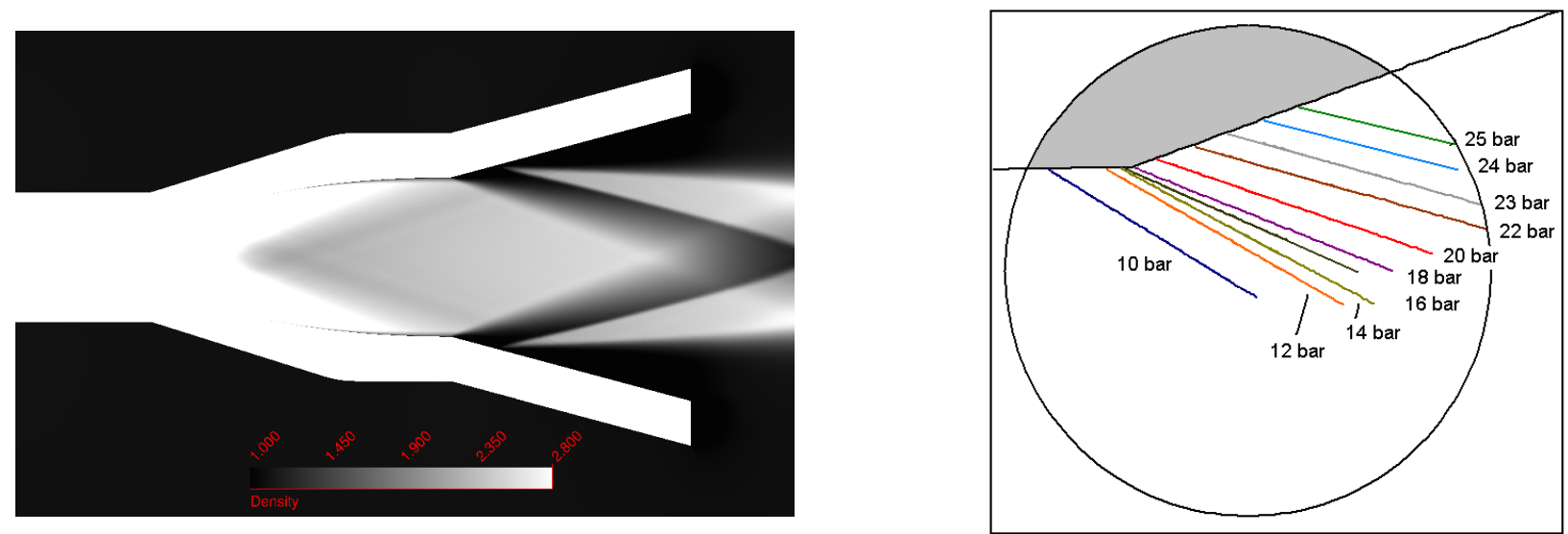

Figure 6. Position and angle of the separation shock obtained with the numerical method.

The density field obtained with the numerical method is also given in Fig. 6 for the cold flow conditions. Similar to the experimental method, the separation shock has been measured out of the flow field. 
The position of the separation point is good predicted as long as it is located in the base nozzle and during sea level mode. For higher NPR values, the numerical method gives a position located further down stream as the actual one. However, when considering the same position of the shock, the angle values are very similar. The experiment can be good simulated with the presented method. A shift in the NPR value has to be taken into account for a correct simulation of the flow in the nozzle model.

\section{V.B. Temperature distribution and heat flux}

Test facility M11 offers only short test duration (up to $60 \mathrm{~s}$ ). Consequently, it is difficult to reach thermal steady state conditions. To circumvent this limitation, the tests were conducted in series with short intervals. Figure 7 illustrates the temperature distribution recorded at the end of a series in altitude mode. The temperatures were recorded in the wall at $1 \mathrm{~mm}$ and $3 \mathrm{~mm}$ from the hot flow side. The temperature decreases progressively along the base nozzle wall. Downstream the contour inflection, the flow is highly expanded, leading to a faster decrease of the wall temperature.

In the same manner, the temperature distribution is given for the numerical method in Fig. 8. At 15 bar, the nozzle flow is in sea level mode, at 20 bar in sneak transition and at 25 and 30 bar, the flow separates further down stream in the inflection. The calculated temperature values are slightly higher than the measured one. This is due to the fact that the calculation supposes steady state conditions, which are not completely reached in the experiment.
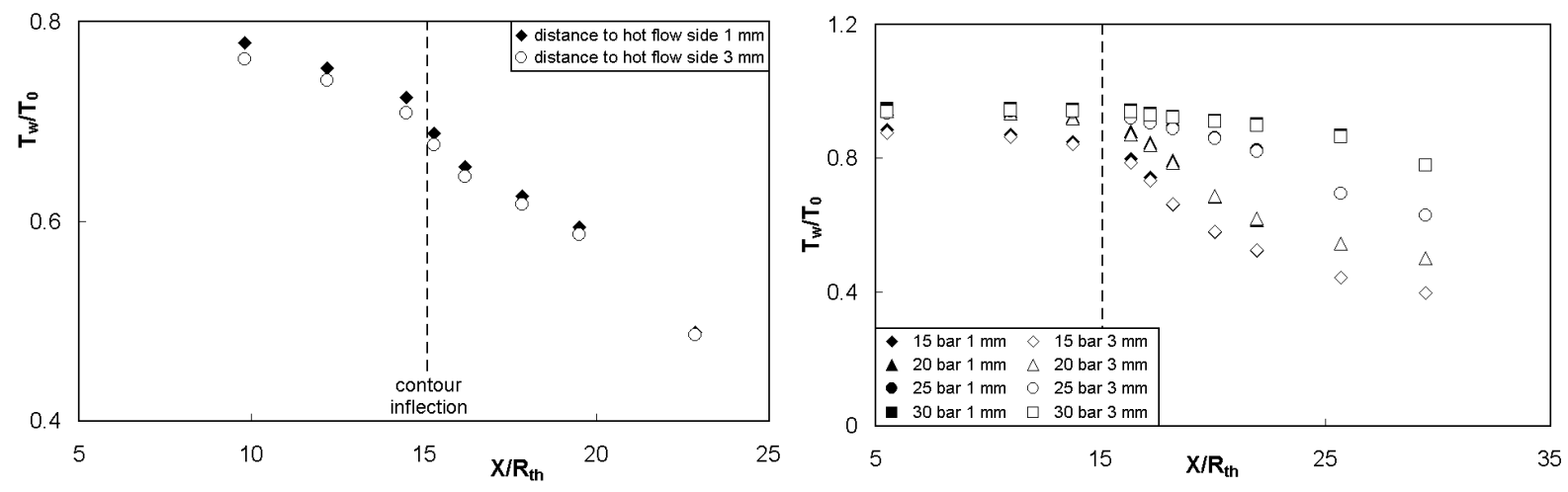

Figure 7. Wall temperature measurements at $1 \mathrm{~mm}$ and Figure 8 . Calculated temperatures at 1 and $3 \mathrm{~mm}$ from $3 \mathrm{~mm}$ from the hot flow side. the hot flow side.

The temperature measurements made in the nozzle wall permit to determine the local heat flux q using the one-dimensional Fourier's law, with $q_{y}=-k d T / d y$. The material conductivity $k$ of the heat resistant steel is $19 \mathrm{~W} \cdot \mathrm{m}^{-1} \cdot \mathrm{K}^{-1}$ in the conditions of test. The distance $d y$ is $2 \mathrm{~mm}$ and corresponds to the radial distance between the position of the thermocouples.

A test case corresponding to the temperature measurements presented in Fig. 7 is chosen. The flow separation is situated in the second part of the inflection $\left(P_{0}=25 \mathrm{bar}\right)$. The temperature repartition is illustrated in Fig. 9 (top) for the flow and the solid body . Figure 9 (bottom) represents the Mach number distribution in the nozzle flow. The separation point corresponds to a temperature increase in the flow.

The local heat flux was then calculated out of the experimental and numerical wall temperatures and is presented in Fig. 10. The same total temperature was set and the total pressure corresponds to 28 bar in the experiment and 25 bar in the simulation. The values of the heat flux are given normalized over the highest value for comparison purposes. Experiment and simulation show a very similar behavior: the heat flux increases in the base nozzle wall, in the vicinity of the contour inflection. It decreases then in the extension wall, with a higher value in the inflection region. In the extension, the numerical method shows an increase in the region where the separation point is located. This effect has already been seen experimentally in cold flow experiments $\left(\mathrm{Stark}^{8}\right)$. The experimental measurements do not show this heat flux increase. However, the reason could be the low density of thermocouples in this area.

The local heat flux has also been calculated for the other flow conditions (sea level mode and sneak transition) and are represented in Fig. 11. Once again, all the values are presented normalized over the first value (corresponding to the axial position of the first temperature measurement point). The three operation modes show a heat flux increase in the vicinity of the inflection. In sea level mode and during sneak transition, this effect is amplified by the temperature increase due to the flow separation either directly 


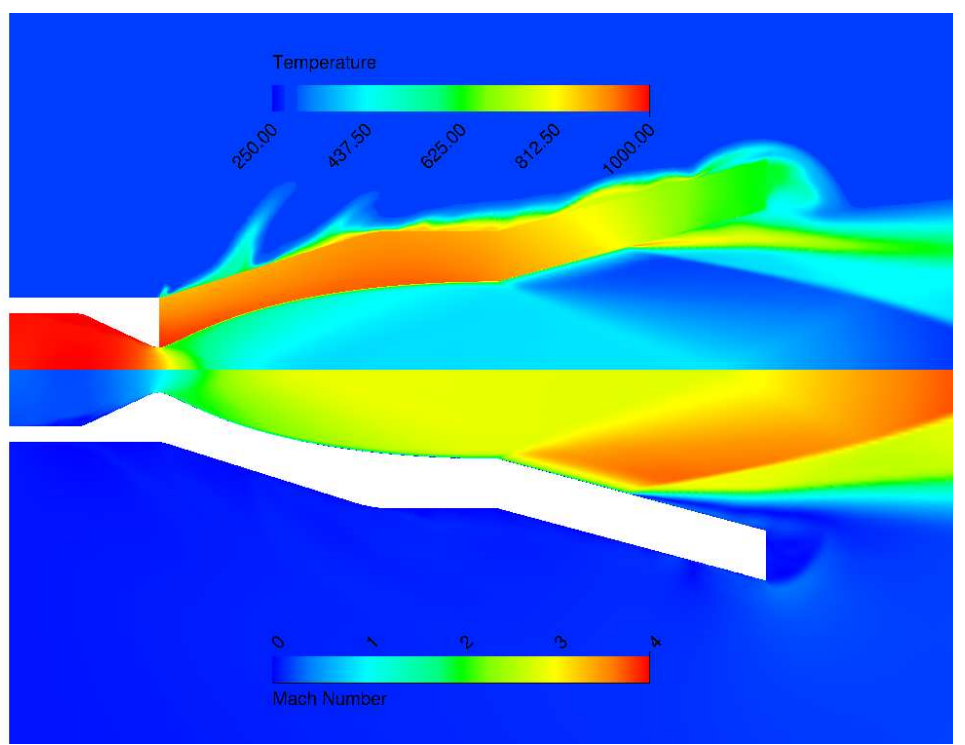

Figure 9. Temperature repartition in the flow and in the nozzle wall (top) and Mach number distribution (bottom) calculated for $P_{0}=25$ bar.

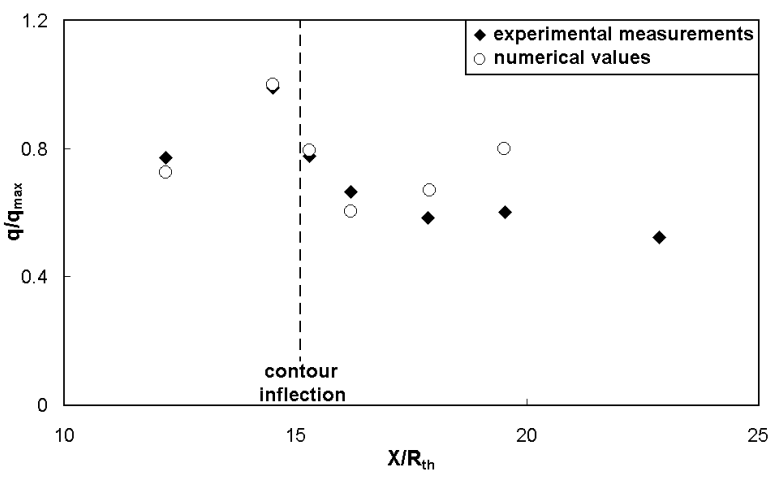

Figure 10. Comparison of experimental and numerical values of the heat flux.

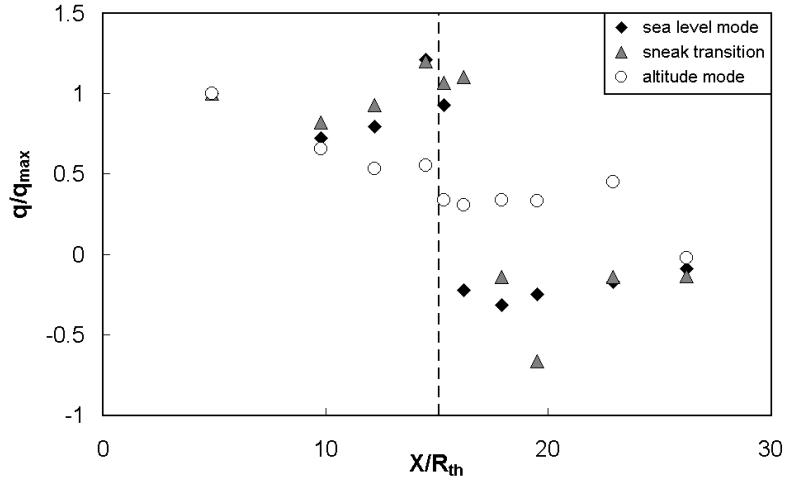

Figure 11. Numerical value of the heat flux in the three nozzle modes.

at the inflection (sea level mode) or in the inflection region (sneak transition). In the extension the flow is separated and the recirculation of ambient air leads to negative values of the heat flux. In altitude mode, the constant pressure extension leads to homogeneous temperature and hence heat flux.

\section{V.C. Thermography}

In addition to the present study on a 2D nozzle model, an axisymmetric nozzle model has been experimentally investigated. The model is represented in Fig. 12 with its geometrical parameters. The tests were conducted under similar conditions at M11 facility. The region of the nozzle throat featured 10 mm wall thickness, the remaining part, from the middle of the base nozzle to the end of the extension, only $3 \mathrm{~mm}$. The thin wall permitted temperature measurements using thermography from the outer side of the nozzle. Thermocouples were also placed in the wall along the nozzle contour.

A picture taken during altitude mode with thermography is given in Fig. 14 (left). The temperature distribution was taken out of the images for the centerline of the nozzle using an in-house tracing program and Matlab. Figure 14 (right) illustrates the temperature distribution along the outer wall obtained with this method in sea level and altitude mode.

For both modes, the wall temperature is high in the base nozzle and decreases along the nozzle wall. In sea level, the temperature decrease starts already up stream to the contour inflection. The flow separates in the extension, leading to a comparatively cold wall $\left(T_{W} / T_{0}=0.4\right)$. In altitude mode, only a minimal 


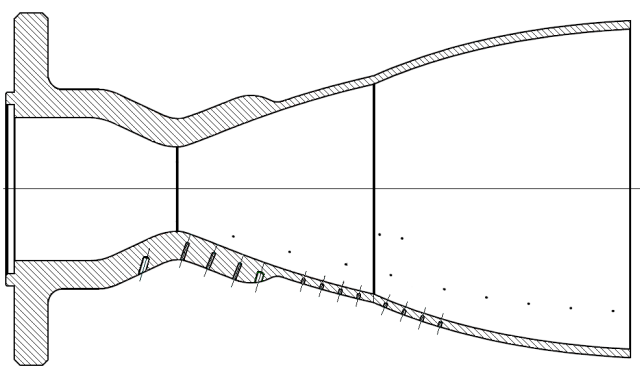

\begin{tabular}{lcc}
\hline Throat radius & $R_{t h}$ & $15 \mathrm{~mm}$ \\
Base length & $L_{b} / R_{t h}$ & 4.7 \\
Extension length & $L_{e} / R_{t h}$ & 10.7 \\
Area ratio & $\epsilon_{b}$ & 6.2 \\
& $\epsilon_{e}$ & 14.3 \\
Inflection angle & $\alpha$ & $11^{\circ}$ \\
\hline
\end{tabular}

Figure 12. Geometry of the axisymmetric dual bell model.

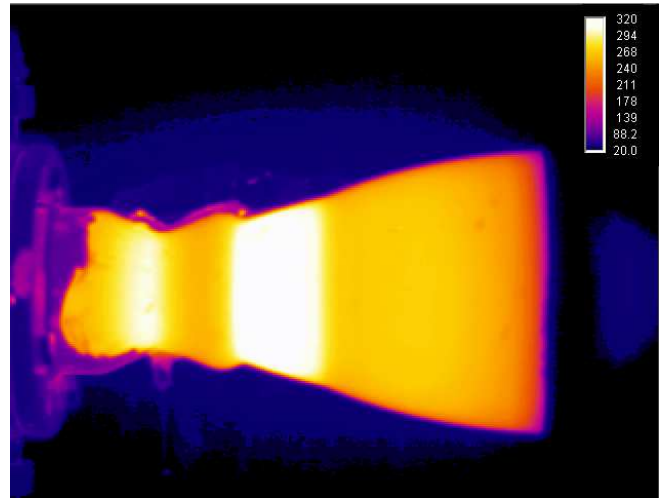

Figure 13. An example of thermal imaging of the nozzle in altitude mode.

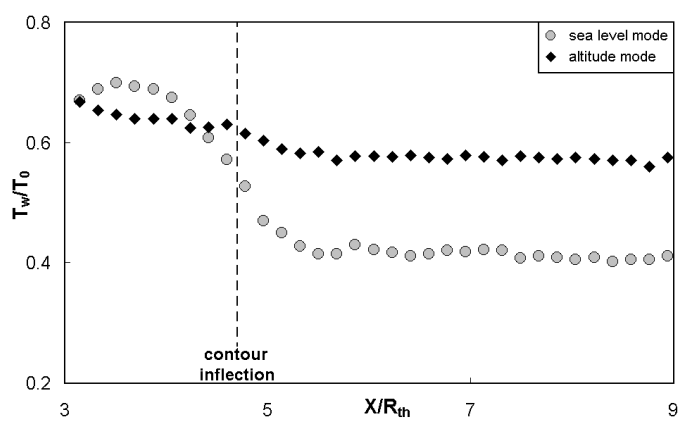

Figure 14. Wall temperature measurements with thermography in sea level and altitude modes.

temperature decrease occurs at the inflection, due to the sudden flow expansion. The wall temperature in the extension is $T_{w} / T_{0}=0.6$.

For the determination of the thermal flux in the nozzle wall, thermocouples were placed in the wall at $1 \mathrm{~mm}$ to the inner side in addition to the thermography. Figure 15 gives representative example of the thermal flux in the axisymmetric nozzle. The same effect as previously seen takes place in the nozzle: the value of the heat flux decreases in the base nozzle, increases in the vicinity of the inflection and decreases again down stream the inflection region. In sea level mode, the flux in the extension is very low till negative.

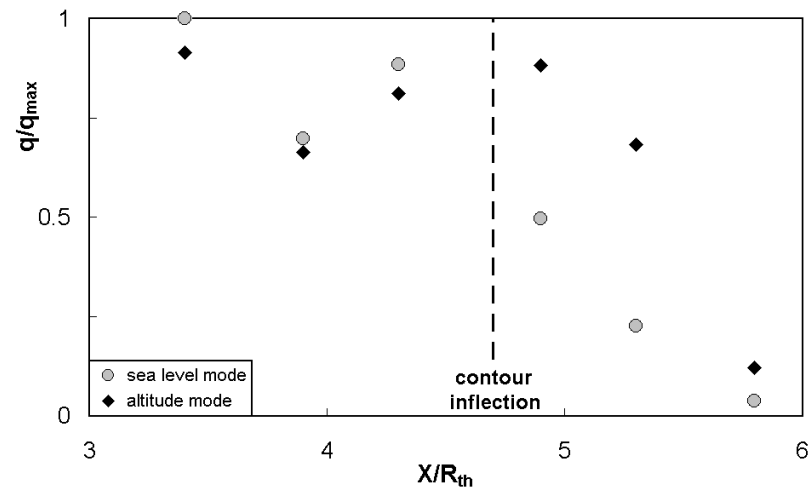

Figure 15. Thermal flux in the axisymmetric nozzle model

The tests conducted in this study were very short (only $15 \mathrm{~s}$ ), so that no thermal steady state could be reached. Besides, the measurement uncertainties using thermography are higher. The consequence is that the experimental results obtained for this campaign feature a significant inaccuracy margin. However, the thermal flux distribution calculated out of the measurements confirms the effect seen for the planar nozzle in classical axisymmetric dual bell nozzles. 


\section{Conclusion}

An experimental study has been conducted on a planar dual bell nozzle for the determination of the thermal flux in the wall. Although the test facility limited the test duration, significant results have been obtained when driving series of tests. The local heat flux determined out of the temperature measurements decreases along the nozzle contour. In the region directly up stream to the contour inflection, the value of the heat flux clearly increase in both operation modes. In altitude mode, the inflection region shows also a heat flux increase. The tests have been recalculated with a numerical method using the commercial code Fluent. The comparison of experiment and calculation shows a very good agreement for the flow and the nozzle wall temperature. The effect of increased heat flux has been confirmed by the numerical method. A first investigation on a sub scale axisymmetric nozzle has shown the same thermal behavior. This effect will have to be taken into account for the design of a proper cooling method for dual bell nozzle in real engine application.

\section{References}

\footnotetext{
${ }^{1}$ Foster, C. and Cowles, F., Experimental study of gas-flow separation in overexpanded exhaust nozzles for rocket motors, Progress Report, 4-103, Jet Propulsion Laboratory, 1949.

${ }^{2}$ Horn, M. and Fisher, S., Dual-Bell Altitude Compensating Nozzles, NASA-CR-194719, 1994.

${ }^{3}$ Hagemann, G., Immich, H., Van Nguyen, T. and Dumnov, G., Advanced Rocket Nozzles, Journal of Propulsion and Power, 1998.

${ }^{4}$ Génin, C., Experimental Study of Flow Behaviour and Thermal Loads in Dual Bell Nozzles, Ph.D. thesis, Université de Valenciennes, France. ISBN 978-3-8322-9230-0, 2010.

${ }^{5}$ Spalart, P. and S. Allmaras, S. A one-equation turbulence model for aerodynamic flows, Technical Report AIAA-92-0439, American Institute of Aeronautics and Astronautics, 1992.

${ }^{6}$ Stark, R. and Hagemann, G., Current status of numerical flow prediction for separated nozzle flows, 2nd European Conference for Aerospace Sciences (EUCASS), Brussels, Belgium, 1-6 July, 2007.

${ }^{7}$ Génin, C. and Stark, R., Hot flow testing of dual bell nozzles, 49th Aerospace Science Meeting, 4-7 January 2011, Orlando, FL, 2011.

${ }^{8}$ Stark, R. and Koschel, W., Experimental Study of the Temperature Rise within the Separation Zone of Bell Type Nozzles, AIAA-2009-5147, 45th AIAA/ASME/SAE/ASEE Joint Propulsion Conference and Exhibit, Denver, Co, 2-5 August, 2009.

${ }^{9}$ Génin, C., Stark, R., Haidn, O., Quering, K. and Frey, M. Experimental and numerical study of dual bell nozzle flow, 4th Europeran Conference for Aerospace Sciences, Saint Petersburg, Russia, 4-8 July 2011.
} 